\title{
Detection of fusion genes at the protein level in leukemia patients via the flow cytometric immunobead assay
}

E. Dekking, PhD ${ }^{\mathrm{a}, \mathrm{b}}$, V.H.J. van der Velden, $\mathrm{PhD}^{\mathrm{a}}$, S. Böttcher, $\mathrm{MD}, \mathrm{PhD}^{\mathrm{c}}$, M. Brüggemann, $\mathrm{MD}, \mathrm{PhD}^{\mathrm{c}}$, E. Sonneveld, $\mathrm{PhD}^{\mathrm{d}}$, A. Koning-Goedheer, BSc ${ }^{\mathrm{d}}$, N. Boeckx, MD, PhD ${ }^{\mathrm{e}}$, P. Lucio, MD, $\mathrm{PhD}^{\mathrm{f}}$, L. Sedek, MSc ${ }^{\mathrm{g}}$, T. Szczepański, MD, $\mathrm{PhD}^{\mathrm{g}}$, T. Kalina, PhD ${ }^{\mathrm{h}}, \mathrm{M}$. Kovac, MSc ${ }^{\mathrm{h}}, \mathrm{P}$. Evans, $\mathrm{PhD}^{\mathrm{i}}$, P.G. Hoogeveen, BSc ${ }^{\mathrm{a}}$, J. Flores-Montero, $\mathrm{MD}, \mathrm{PhD}^{\mathrm{j}}, \mathrm{A}$. Orfao, $\mathrm{MD}, \mathrm{PhD}^{\mathrm{j}}$, W.M. Comans-Bitter, BSc ${ }^{\text {a }}$, F.J.T. Staal, $\mathrm{PhD}^{\mathrm{a}}$, J.J.M. van Dongen, MD, PhD, Professor ${ }^{\mathrm{a}, *}$ On behalf of the EuroFlow Consortium (EU-FP6, LSHB-CT-2006-018708)

a Department of Immunology, Erasmus MC, Rotterdam, The Netherlands

${ }^{\mathrm{b}}$ Dynomics, Rotterdam, The Netherlands

' 2 nd Department of Medicine, University Hospital Schleswig-Holstein, Kiel, DE, Germany

${ }^{\mathrm{d}}$ Dutch Childhood Oncology Group (DCOG), The Hague, The Netherlands

${ }^{\mathrm{e}}$ Department of Laboratory Medicine, Hematology, University Hospitals Leuven, Belgium

${ }^{\mathrm{f}}$ Department of Hematology, Instituto Portugues de Oncologia, Lisbon, Portugal

${ }^{\mathrm{g}}$ Department of Pediatric Hematology and Oncology, Medical University of Silesia, Zabrze, Poland

${ }^{\mathrm{h}}$ Department of Pediatric Hematology and Oncology, Charles University, Prague, Czech Republic

${ }^{i}$ Department of Haematology Malignancy, St. James University Hospital, Leeds, UK

${ }^{\mathrm{j}}$ Department of Medicine, Cancer Research Centre (IBMCC-CSIC-USAL) and Cytometry Service, University of Salamanca, Spain

Keywords:

fusion gene

fusion protein

immunobead assay

BCR-ABL

PML-RARA
Nowadays, the presence of specific genetic aberrations is progressively used for classification and treatment stratification, because acute leukemias with the same oncogenetic aberration generally form a clinically and diagnostically homogenous disease entity with comparable prognosis. Many oncogenetic aberrations in acute leukemias result in a fusion gene, which is transcribed into fusion transcripts and translated into fusion proteins, which are assumed to play a critical role in the oncogenetic process. Fusion gene aberrations are detected by karyotyping, FISH, or RT-PCR analysis. However, these molecular genetic techniques are laborious and time consuming, which is in contrast to flow cytometric techniques. Therefore we developed a flow cytometric

\footnotetext{
* Corresponding author. Dept. of Immunology, Erasmus MC, University Medical Center Rotterdam, Dr. Molewaterplein 50, 3015 GE Rotterdam, The Netherlands. Tel.: +31 107044094.

E-mail address: j.j.m.vandongen@erasmusmc.nl (J.J.M. van Dongen).
} 
immunobead assay for detection of fusion proteins in lysates of leukemia cell samples by use of a bead-bound catching antibody against one side of the fusion protein and fluorochrome-conjugated detection antibody. So far, we have been able to design such fusion protein immunobead assays for BCR-ABL, PML-RARA, TELAML1, E2A-PBX1, MLL-AF4, AML1-ETO and CBFB-MYH11. The immunobead assay for detection of fusion proteins can be performed within 3 to 4 hours in a routine diagnostic setting, without the need of special equipment other than a flow cytometer. The novel immunobead assay will enable fast and easy classification of acute leukemia patients that express fusion proteins. Such patients can be included at an early stage in the right treatment protocols, much faster than by use of current molecular techniques. The immunobead assay can be run in parallel to routine immunophenotyping and is particularly attractive for clinical settings without direct access to molecular diagnostics.

(C) 2010 Published by Elsevier Ltd.

\section{Introduction}

Nowadays, the diagnosis and classification of hematological malignancies is no longer based only on cytological and histomorphological characteristics or the immunophenotype of the malignant cells. Progressively, also the presence of specific genetic aberrations is used for classification and treatment stratification, because malignancies with the same oncogenetic aberration generally form a clinically and diagnostically homogeneous disease entity with comparable prognosis [1-3].

In leukemias, many chromosome aberrations result in an aberrant fusion gene, caused by the incorrect coupling of the upstream part of one gene to the downstream part of another gene and vice versa (reciprocal fusion gene). In most leukemias, such fusion genes are transcribed into fusion transcripts, which are translated into fusion proteins which (at least in part) have been shown to play a critical role in the pathogenesis of the leukemias. The first chromosome aberration discovered in leukemia patients was the so-called Philadelphia $(\mathrm{Ph})$ chromosome, which was shown to result from the $(9 ; 22)$ translocation, in which the $B C R$ gene is aberrantly coupled to the $A B L 1$ gene $[4,5]$. The $t(9 ; 22)$ is found in virtually all patients with chronic myeloid leukemia (CML) and in a fraction of the patients with B-cell precursor acute lymphoblastic leukemia (BCP-ALL), particularly in adults [6].

In BCP-ALL, also other fusion genes have been discovered such as $t(1 ; 19)$ with the E2A-PBX1 fusion gene, $\mathrm{t}(4 ; 11)$ with the MLL-AF4 fusion gene, and $\mathrm{t}(12 ; 21)$ with the TEL-AML1 fusion gene. In patients with acute myeloid leukemia (AML), several types of fusion gene aberrations have been found; the most frequently occurring aberrations are $\mathrm{t}(8 ; 21)$ with the AML1-ETO gene and inv(16) or $\mathrm{t}(16 ; 16)$ with the CBFB-MYH11 gene $[2,7]$. The $t(15 ; 17)$ with the PML-RARA fusion gene is found in virtually all $(>95 \%)$ patients with acute promyelocytic leukemia (APL) [8].

The occurrence and relative frequency of fusion gene aberrations not only depends on the type of leukemia, but also on the age of the patient. Particularly in BCP-ALL, large differences in relative frequencies are found between different age groups (Fig. 1) [9]. For example, $M L L$ fusion genes, such as $M L L-A F 4$, occur at high frequency in infant ALL ( $~ 80 \%)$, whereas the $B C R-A B L$ fusion gene is rare at young age $(<2 \%)$ and its frequency rapidly increases with age (Fig. 1) [9].

Most chromosome aberrations have prognostic value for treatment outcome. TEL-AML1 in childhood BCP-ALL and AML1-ETO or CBFB-MYH11 in AML are associated with a good prognosis. In contrast, most MLL gene aberrations as well as the BCR-ABL1 fusion gene in BCP-ALL are associated with poor prognosis, and the afflicted patients are being treated on high-risk protocols [2,9].

When it became clear that the fusion gene products contribute to the malignant transformation process, several pharmaceutical research programs focused on the development of drugs to block the fusion proteins in their function. For example, the BCR-ABL fusion protein induces increased signaling activation via the ABL tyrosine kinase domain, which appears to result in higher proliferation and 


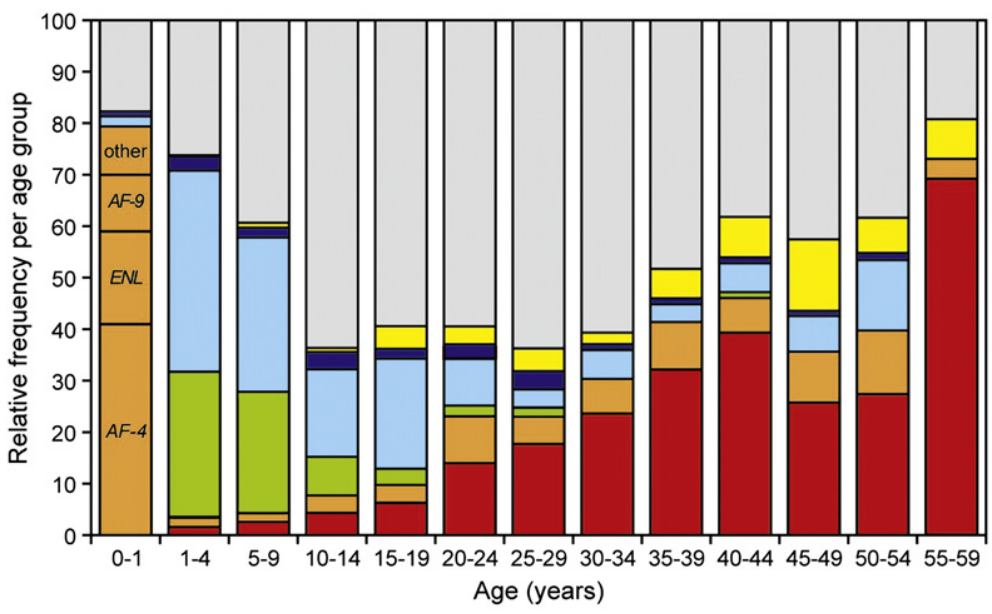

$\square_{\mathrm{t}(4 ; 1) / 11923} \square_{\mathrm{t}(12 ; 21)} \square_{\mathrm{HeH}}$

$\mathrm{t}(1 ; 19)$

$14 q 32$

other

Fig. 1. Relative distribution of different types of chromosome aberrations in BCP-ALL patients in different age groups. The MLL gene aberrations dominate in infant $\operatorname{ALL}(\sim 80 \%), \mathrm{t}(12 ; 21)$ with the TEL-AML1 fusion gene dominates in childhood ALL, whereas $\mathrm{t}(9 ; 22)$ with the $B C R-A B L$ fusion gene progressively increases from $2 \%$ in young children to $60-70 \%$ in older patients (see Szczepañski et al. in Ref. [9]).

survival of the leukemia cells [10]. A decade ago, the first specific tyrosine kinase inhibitor (TKI), imatinib, was identified, which blocks the signaling function of the ABL tyrosine kinase domain [11,12]. In the meantime, several different TKI's have been developed, which differ in their blocking mechanisms [13]. Another example for targeted therapy is the use of retinoid differentiating agents such as all-trans retinoid acid (ATRA) in APL patients with the PML-RARA fusion gene [14,15]. ATRA binds to one of the RARA domains, thereby blocking the PML-RARA fusion protein in its aberrant function of inhibiting the maturation of APL cells. The introduction of TKIs in CML and of ATRA in APL has dramatically improved the clinical outcome in both diseases.

Consequently, both for reasons of prognostic classification and for reasons of specifically targeted treatment options, it has become increasingly important to have a fast and simple, though accurate assay for the detection of fusion genes.

\section{Detection of chromosome aberrations with fusion genes}

To date, the various types of oncogenetic aberrations are detected at the chromosome level by means of karyotyping and fluorescence in situ hybridization (FISH), or at the mRNA/transcript level by RT-PCR analysis $[7,16,17]$. The availability of these diagnostic techniques is generally restricted to large references centers, which have the appropriate laboratory facilities (such as culture rooms and PCR facilities) as well as well-trained technicians. Furthermore, the (molecular) genetic techniques are time consuming: 1-2 days for FISH and PCR techniques and even 1-2 weeks for karyotyping $[7,16,17]$. Nevertheless, it is important to provide a fast and reliable diagnosis to support efficient clinical management and to assign patients to the appropriate treatment protocols quickly after leukemia diagnosis.

\section{Detection of fusion proteins}

Antibody-based techniques for the detection of normal and aberrant proteins are much faster than molecular genetic techniques, generally give different and extra information, and can be performed more easily without special laboratory facilities. Furthermore, fusion proteins, by definition, have a new tumor-specific fusion epitope, i.e. the coupling site of the two protein fragments, each of which originally derived from a different gene. Therefore, we tried hard to design specific antibodies against fusion epitopes of several fusion proteins, particularly against the fusion epitopes of the different BCR- 


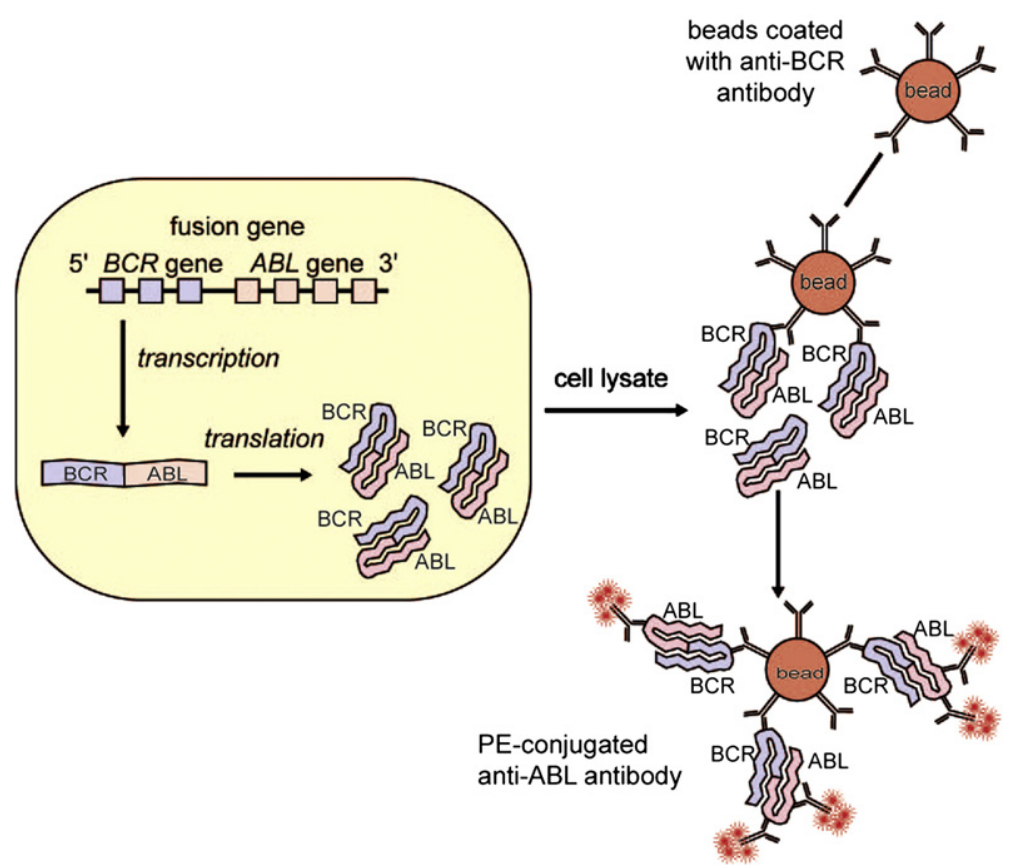

Fig. 2. Principle of flow cytometric BCR-ABL immunobead assay. Fusion of the upstream part of the $B C R$ gene to the downstream part of the $A B L$ gene leads to the generation of an oncogenic BCR-ABL protein. Upon release of the fusion proteins by lysis of the leukemic cells, they are recognized by both an anti-BCR antibody coupled to a bead and a PE-labeled anti-ABL antibody. The beads are evaluated on a flow cytometer, which can detect the PE fluorescence signal. If only normal BCR and ABL proteins are present, no PE fluorescence signal will be detected on the beads.

ABL fusion proteins. This turned out to be far more difficult than anticipated. We succeeded in developing polyclonal rabbit antisera against two BCR-ABL fusion epitopes [18-20] and a murine monoclonal antibody was designed against a single BCR-ABL fusion epitope [21]. However, all obtained antibodies worked solely under denaturing conditions, such as Western blotting, and none of them was suited for intracellular staining in microscopy or flow cytometry.

Consequently, we decided to develop another antibody-based technique, which does not target the fusion epitope itself but the two parts of the fusion protein via a catching antibody against one part of the fusion protein and a labelled detection antibody against the other part of the fusion protein [22]. Initially, a "dipstick" approach was used, in which the catching antibody was linked to a membrane [23]. This approach worked reliably, but was too slow and too time-consuming for routine diagnostic applications. Therefore, we modified this technique and created a faster and easier flow cytometric technique, wherein the catching antibody is coupled to a bead and the detection antibody is conjugated with a fluorochrome (Fig. 2) [24]. We anticipated that such immunobead assay would be able to detect a fusion protein in lysates of leukemic cells, provided that correct antibodies were available. However, virtually all commercially available antibodies against various domains of the classical fusion proteins appeared not to be suited for application in the immunobead assay, particularly because of non-specific binding to other proteins (cross-reactivity) and because of insufficient affinity. Therefore, we decided to design new antibodies for the immunobead-based detection of the most common fusion proteins in BCP-ALL, AML and CML.

\section{Immunobead assay for the detection of BCR-ABL fusion proteins}

The $t(9 ; 22)$ with the $B C R-A B L$ fusion gene can present in several variants, because multiple different breakpoint cluster regions (bcr's) occur in the BCR gene (Fig. 3A). The first identified bcr was the socalled "major $b c r$ " (M-bcr), which is predominantly involved in CML patients and results in the BCR-ABL 
A

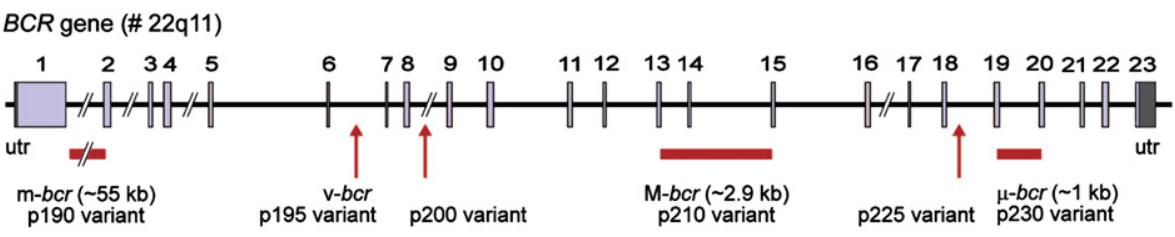

ABL gene (\# 9q34)

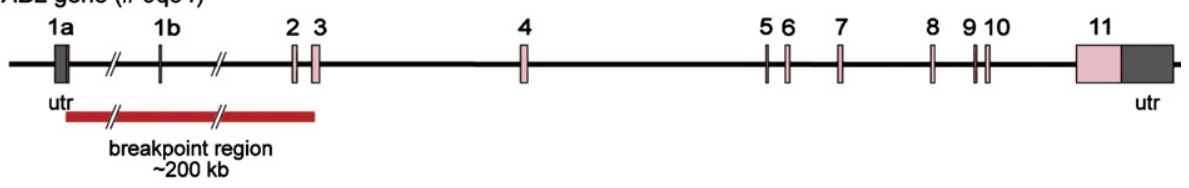

B

m-bcr, p190 proteins

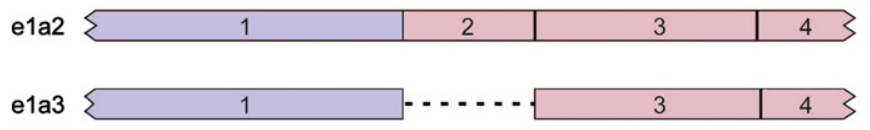

M-bcr, p210 proteins

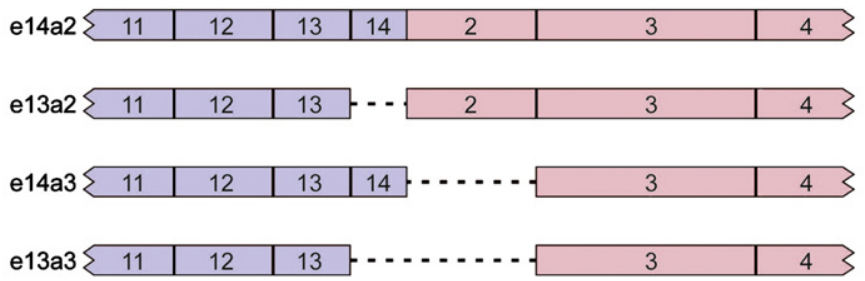

$\mu-b c r$, p230 proteins

\begin{tabular}{|c|c|c|c|c|c|c|}
\hline $\mathrm{e} 19 \mathrm{a} 2<$ & 17 & 18 & 19 & 2 & 3 & 4 \\
\hline e19a3 & 17 & 18 & 19 & & 3 & 4 \\
\hline
\end{tabular}

\section{rare variants}

$\mathrm{v}-b c r, \mathrm{p} 195$ proteins

e6a2

\begin{tabular}{|l|l|l|l|l|}
\hline 5 & 6 & 2 & 3 & 4 \\
\hline
\end{tabular}

p200 proteins

e8a2

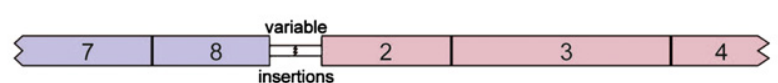

p225 proteins

e18a2

\begin{tabular}{|l|l|l|l|l|}
\hline 17 & 18 & 2 & 3 & 4 \\
\hline
\end{tabular}

Fig. 3. Structure of the $B C R$ and $A B L$ genes with breakpoint regions and corresponding fusion gene transcripts. A. The $A B L$ gene contains one large breakpoint region ( $\sim 200 \mathrm{~kb}$ ), whereas three breakpoint regions have been found in the $B C R$ gene: $\mathrm{m}-b c r, \mathrm{M}-b c r$, and $\mu-b c r$, which are associated with the p190, p210, and p230 BCR-ABL fusion proteins, respectively.[4-6, 25-27] In addition, several rare breakpoints have been reported (see arrows), including the v-bcr breakpoint. $[26,28,30] \mathrm{B}$. The three well-defined breakpoint regions in the $B C R$ gene can produce at least eight different fusion transcripts, because of alternative splicing in the $A B L$ gene (splicing to exon 2 or exon 3) and, because the M-bcr consists of two intronic regions (intron 13 and intron 14) [26]. Additional BCR-ABL fusion transcripts have been described, but they are extremely rare or, to date, have been reported only once $[26,28,30]$.

p210 fusion protein. Later, the so-called "minor $b c r$ " (m-bcr) was identified, occurring mostly in adult BCP-ALL patients and resulting in the BCR-ABL p190 fusion protein (Table 1) [4-7]. In addition to these two common breakpoints, several less frequent breakpoint variants have been found, such as $\mu-b c r, v-$ $b c r$, etc. (Fig. 3A) [25-28]. The different breakpoints result in different fusion transcripts and, 
Table 1

Most frequent chromosome aberrations with fusion genes in leukemia.

\begin{tabular}{|c|c|c|c|c|c|c|c|}
\hline \multirow{3}{*}{$\begin{array}{l}\text { Chromosome } \\
\text { aberration }\end{array}$} & \multirow[t]{3}{*}{ Fusion genes } & \multicolumn{2}{|l|}{ BCP-ALL } & \multicolumn{3}{|l|}{ AML } & \multirow[t]{3}{*}{$\mathrm{CML}$} \\
\hline & & \multirow[t]{2}{*}{ Children } & \multirow[t]{2}{*}{ Adults } & \multirow[t]{2}{*}{ Children } & \multicolumn{2}{|l|}{ Adults } & \\
\hline & & & & & $\leq 60 \mathrm{y}$ & $>60 y$ & \\
\hline $\mathrm{t}(1 ; 19)(\mathrm{q} 23 ; \mathrm{p} 13)$ & $E 2 A-P B X 1$ & $3-5 \%$ & $2-4 \%$ & - & - & - & - \\
\hline $11 \mathrm{q} 23$ aberrations & MLL fusions & $4-5 \%$ & $5-6 \%$ & $8-10 \%$ & $\sim 10 \%$ & $\sim 10 \%$ & - \\
\hline $\mathrm{t}(4 ; 11)(\mathrm{q} 21 ; \mathrm{q} 23)$ & $M L L-A F 4$ & $3-4 \%^{\mathrm{a}}$ & $4-8 \%$ & $<1 \%$ & $<1 \%$ & $<1 \%$ & - \\
\hline \multirow[t]{2}{*}{$\mathrm{t}(9 ; 22)(\mathrm{q} 34 ; \mathrm{q} 11)$} & BCR-ABL $p 190$ & $2-4 \%$ & $10-25 \%{ }^{b}$ & $<1 \%$ & $<1 \%$ & $<1 \%$ & $<5 \%$ \\
\hline & $B C R-A B L$ p 210 & $1-2 \%$ & $5-15 \%$ & $<1 \%$ & $<1 \%$ & $<1 \%$ & $\pm 95 \%$ \\
\hline $\mathrm{t}(12 ; 21)(\mathrm{p} 13 ; \mathrm{q} 22)$ & TEL-AML1 & $20-25 \%$ & $<2 \%$ & - & - & - & - \\
\hline $\mathrm{t}(15 ; 17)(\mathrm{q} 22 ; \mathrm{q} 21)$ & $P M L-R A R A$ & - & - & $8-10 \%^{\mathrm{c}}$ & $5-15 \%$ & $2-6 \% c$ & - \\
\hline $\mathrm{t}(8 ; 21)(\mathrm{q} 22 ; \mathrm{q} 22)$ & AML1-ETO & - & - & $10-14 \%$ & $6-8 \%$ & $2-3 \%$ & - \\
\hline \multirow[t]{2}{*}{$\operatorname{inv}(16)(p 13 ; q 22)$} & CBFB-MYH11 & - & - & $5-7 \%$ & $5-6 \%$ & $3-4 \%$ & - \\
\hline & TOTAL & $30-35 \%$ & $35-45 \%$ & $30-35 \%$ & $25-30 \%$ & $15-20 \%$ & $>98 \%$ \\
\hline
\end{tabular}

\footnotetext{
${ }^{a}$ In infants ( $<1$ year) with ALL, the frequency of $M L L$ gene aberrations and $\mathrm{t}(4 ; 11)$ is approximately $80 \%$ and $40 \%$, respectively (see also Fig. 1 and Table 3).

$\mathrm{b}$ In the group of elderly BCP-ALL patients, the percentage of BCR-ABL positivity increases to $60-70 \%$ (Fig. 1).

${ }^{c}$ In Southern Europe (ES, PT, FR and IT), the relative frequency of $\mathrm{t}(15 ; 17)$ with the PML-RARA fusion gene is much higher (up to $25-30 \%$ of $A M L)$.
}

consequently, in different fusion proteins (Fig. 3B). The traditional PCR-based diagnostics use primers which mainly detect the M-bcr and m-bcr variants, implying that the transcripts of other $B C R-A B L$ fusion gene variants might well be missed [7,17,27].

To ensure the recognition of all variants of the BCR-ABL fusion proteins, we designed the anti-BCR antibody against a unique epitope upstream of all $B C R$ gene breakpoints, i.e. in $B C R$-exon-1. Analogously, the anti-ABL antibody recognized an epitope downstream of the large $A B L$ breakpoint region, i.e. in the ABL-SH2 domain. By using these two antibodies against "outer" epitopes, the BCR-ABL immunobead assay was expected to recognize all types of BCR-ABL fusion proteins, independent of the breakpoint position $[29,30]$.

Initial testing of BCR-ABL positive cell lines and patient materials showed good results when the anti-BCR antibody was used as catching antibody (bound to the bead) and the anti-ABL antibody as detection antibody, conjugated with phycoerythrin (PE). However, when we used lysates from mature myeloid cells, the fluorescent signals in the BCR-ABL immunobead assay were clearly weaker than expected [29]. Additional experiments showed that the lower signals were caused by protein degradation via protease activity from the granules in more mature myeloid cells, such as granulocytes as well as CML cells. Upon lysis of these myeloid cells, sufficiently high levels of proteases were released to digest the proteins in the cell lysate, including the BCR-ABL fusion proteins. Multiple experiments with several types of protease inhibitors showed that it was critically important to add a mixture of different types of protease inhibitors to the various incubation steps of the immunobead assay. This modification of the assay reduced the protein degradation substantially. However, delayed processing of CML samples might still result in some protein degradation. Therefore, it is advised to perform the BCR-ABL immunobead assay, particularly in cells from chronic phase CML, within 24$36 \mathrm{~h}$ after sampling [29].

The EuroFlow Consortium evaluated a series of 145 patient samples including 19 CML, 78 BCP-ALL, 18 T-lineage ALL, 27 AML as well as 72 healthy donors. The results from the BCR-ABL immunobead assay were completely concordant (100\%) with the RQ-PCR analysis of $B C R$ - $A B L$ transcripts (Fig. 4) [29]. Importantly, the BCR-ABL immunobead assay detected the fusion proteins of all types of $B C R$ breakpoints: p190, p210, p225, and p230 [29,30].

Dilution experiments were performed for sensitivity testing. Three different BCR-ABL positive cell lines were diluted into blood mononuclear cells (MNC) or into white blood cells (WBC, i.e. including granulocytes). These dilution experiments showed good sensitivities of at least $1 \%$ (Fig. 5) [29]. 


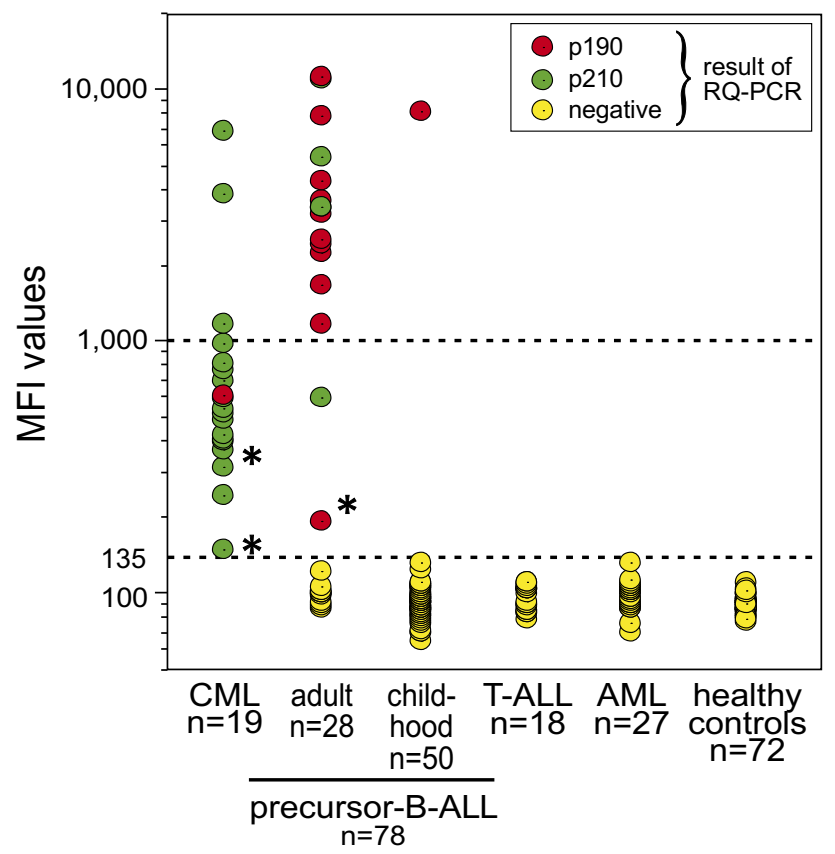

Fig. 4. Results of the EuroFlow testing of the BCR-ABL immunobead assay. The MFI values are plotted per patient group (see insert for symbols). The BCR-ABL positive BCP-ALL patients showed significantly higher BCR-ABL protein expression levels than CML patients ( $p<0.001$ in Mann-Whitney $U$ test). * Repeated analysis for confirmation of weak positivity (see; Weerkamp et al., 2009 , Ref. [29]).

\section{Immunobead assay for the detection of PML-RARA fusion proteins}

Since the first description of APL, the life-threatening bleeding problems have been identified as the most notorious manifestation of the disease, requiring instant treatment [14,15,31]. Many hematologists consider the correct diagnosis in a patient with APL as a medical emergency, because of the

A

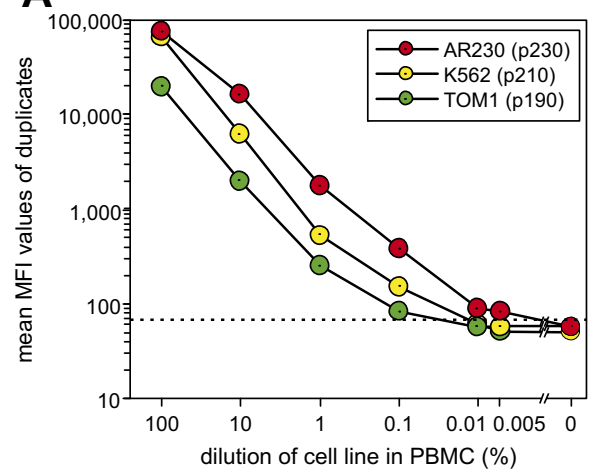

B

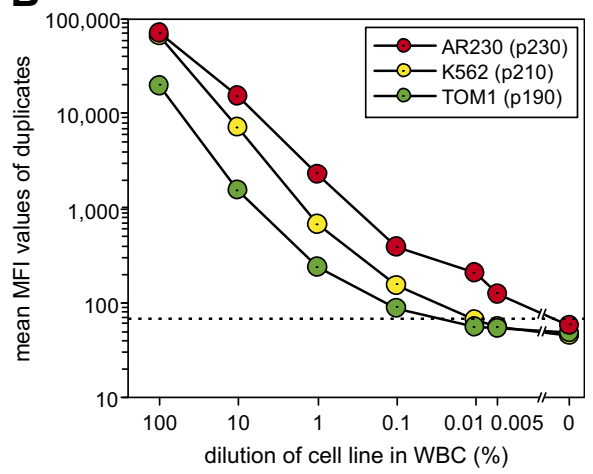

Fig. 5. Sensitivity testing of the BCR-ABL immunobead assay in dilution experiments with cell lines. Three cell lines with different BCR-ABL protein variants were diluted in PBMC (panel A) or WBC (panel B). Duplicate experiments (mean MFI values in graph) showed that AR230 with p230 and K562 with p210 gave good sensitivities of at least $0.1 \%$, whereas TOM- 1 with p190 reached a sensitivity of at least $1 \%$. The dotted line represents the mean MFI value plus 3SD of the six negative samples (PBMC or WBC); this value is lower than in Fig. 4 because for this set of experiments, instrument settings were not based on the CS\&T beads (see: Weerkamp et al., 2009, Ref. [29]). 
A
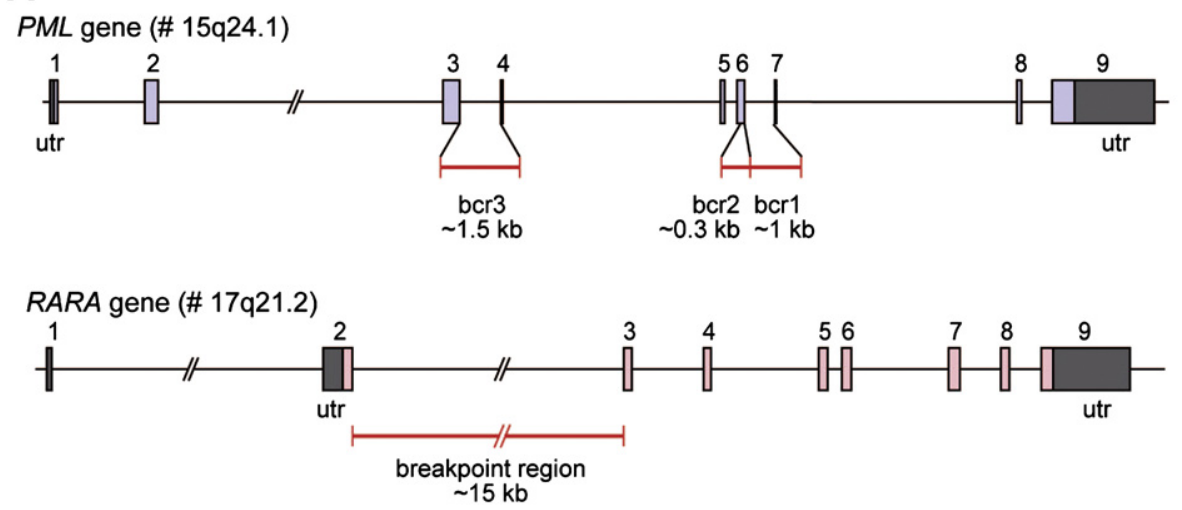

B

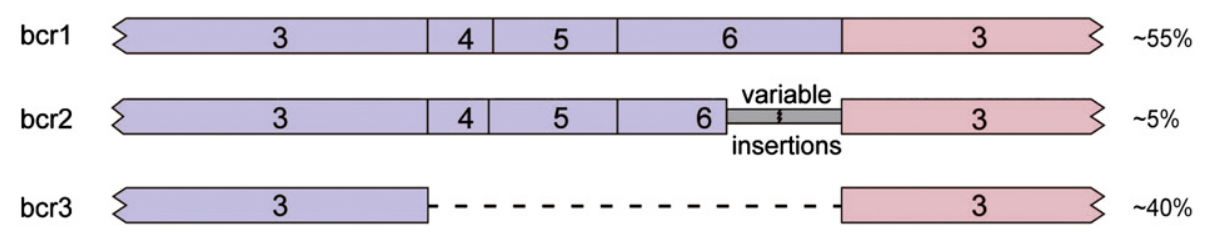

Fig. 6. Structure of the $P M L$ and RARA genes with the breakpoint regions and the corresponding fusion gene transcripts. A. The $P M L$ gene contains three well-defined small bcr's: bcr1 in intron 6, bcr2 in the downstream part of exon 6 , and bcr3 in intron 3 . In the $R A R A$ gene, the breakpoints cluster in intron $2(\sim 15 \mathrm{~kb})$. B. The three bcr's in the PML gene result in three different PML-RARA fusion transcripts.

consequences of the disseminated coagulopathy, which remains the major cause of death in APL patients. Consequently, a positive diagnosis of APL is of utmost importance for patient care. Although the clinical and cytomorphological picture of APL seems relatively clear, the leukemic cells in a subset of patients (5-10\%) do not have the typical APL morphology [32]. Consequently, it is strongly recommended to make a fast and accurate diagnosis of the PML-RARA aberration. Because of the risk of early death due to bleeding, it is recommended that the APL diagnosis be excluded in each patient with newly diagnosed AML [14]. Such diagnosis should preferable be provided within hours, not within days!

The vast majority $(97 \%)$ of APL patients have the $(15 ; 17)$ or a variant translocation, resulting in the PML-RARA fusion gene [8]. The breakpoints in the RARA gene are all located in intron 2, whereas virtually all $P M L$ gene breakpoints cluster in three bcr's: intron 6 ( $b c r 1 ; \sim 55 \%$ of cases), exon 6 ( $b c r 2$; $\sim 5 \%$ of cases), or intron 3 (bcr3; $\sim 40 \%$ of cases), resulting in three major types of PML-RARA fusion transcripts (Fig. 6) [7,17].

For the PML-RARA immunobead assay, the anti-RARA antibody was designed against protein sequences downstream of the RARA breakpoint and used as catching antibody, linked to the bead. The anti-PML antibody was designed against an exposed amino acid sequence, which was not homologous to other proteins and located upstream of all three $b c r$ 's; the anti-PML antibody was PE conjugated and used as detection antibody (Fig. 6). This PML-RARA immunobead assay showed good results in PMLRARA positive cell lines as well as in a small series of APL patients (E. Dekking et al, manuscript in preparation).

Subsequently, the EuroFlow Consortium tested the prototype PML-RARA immunobead assay in 162 acute leukemias, including 46 APL, 66 other AML, 34 BCP-ALL, and 16 T-lineage ALL. The results of the PML-RARA immunobead assay and of the PCR analysis of PML-RARA transcripts were fully concordant. Importantly, the PML-RARA fusion proteins of all three $b c r$ types were detectable by the immunobead assay (E. Dekking et al, manuscript in preparation). 
Table 2

Development of immunobead assays for detection of fusion proteins.

\begin{tabular}{|c|c|c|}
\hline Leukemia & Fusion protein & Diagnostic evaluation \\
\hline CML and BCP-ALL & BCR-ABL & RUO kit tested and approved (now commercially available) ${ }^{\mathrm{a}}$ \\
\hline \multirow[t]{3}{*}{ BCP-ALL } & TEL-AML & $\begin{array}{l}\text { All three immunobeads work in "singleplex" format; } \\
\text { the BCP-ALL multiplex tube is close to completion }\end{array}$ \\
\hline & E2A-PBX2 & \\
\hline & MLL-AF4 & \\
\hline \multirow[t]{2}{*}{ AML } & AML-ETO & $\begin{array}{l}\text { Prototype AML "Core-binding-factor" tube is completed } \\
\text { and shows good performance }{ }^{\mathrm{a}}\end{array}$ \\
\hline & CBFB-MYH11 & \\
\hline AML/APL & PML-RARA & 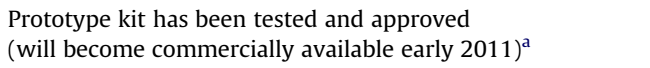 \\
\hline
\end{tabular}

a The BCR-ABL RUO kit is available via BD Biosciences (San José, CA). Also the AML “Core-binding-factor" kit and the PML-RARA kit will become available via BD Biosciences.

\section{Immunobead assays for other fusion proteins}

In the development of fusion protein assays, we gave priority to the BCR-ABL and PML-RARA immunobeads, because of their diagnostic, prognostic, and therapeutic importance. In addition, antibodies have been designed and selected for the other five most frequently occurring fusion proteins: TEL-AML1, E2A-PBX1, and MLL-AF4 in BCP-ALL as well as AML1-ETO and CBFB-MYH11 in AML.

All immunobead assays show good to very good results in the "singleplex" format, in which all incubation and lysis conditions have been optimized for the involved fusion protein. In this context, it should be noted that some fusion proteins are mainly located in the cytoplasm, whereas others are mainly located in the nucleus. This implies that in some cases release of nuclear fusion protein is required by lysing not only the cell membrane but also the nuclear membrane. This needs a more intensive lysis procedure, which might not be suited for each fusion protein.

One of the typical advantages of immunobead assays is the possibility of multiplexing, whereby the combined immunobeads differ in size, color, or other characteristics. In line with this possibility, we planned to design multiplex immunobead assays for detecting the different types of fusion proteins per disease category, for example BCP-ALL and AML. Consequently, we worked on the composition of a BCP-ALL multiplex tube for TEL-AML1, E2A-PBX1, and MLL-AF4, and a multiplex AML Core Binding

Table 3

Frequency of various types of $M L L$ fusion genes in acute leukemias. ${ }^{a}$

\begin{tabular}{|c|c|c|c|c|c|c|}
\hline \multirow[t]{2}{*}{ MLL translocation } & \multicolumn{3}{|l|}{ ALL } & \multicolumn{2}{|l|}{ AML } & \multirow[t]{2}{*}{ All acute leukemias } \\
\hline & Infants & Children & Adults & Children & Adults & \\
\hline$M L L^{+\mathrm{b}}$ & $\sim 80 \%$ & $4-5 \%$ & $5-6 \%$ & $8-10 \%$ & $\sim 10 \%$ & $\sim 10 \%$ \\
\hline$M L L-A F 4^{\mathrm{C}}$ & $\sim 52 \%$ & $\sim 46 \%$ & $\sim 85 \%$ & $<1 \%$ & $<1 \%$ & $\sim 42 \%$ \\
\hline MLL-AF9 & $\sim 13 \%$ & $\sim 16 \%$ & $\sim 2 \%$ & $\sim 32 \%$ & $\sim 29 \%$ & $\sim 16 \%$ \\
\hline$M L L-E N L$ & $\sim 22 \%$ & $\sim 21 \%$ & $\sim 9 \%$ & $\sim 3 \%$ & $\sim 8 \%$ & $\sim 11 \%$ \\
\hline MLL-AF10 & $\sim 3 \%$ & $\sim 5 \%$ & $<1 \%$ & $\sim 23 \%$ & $\sim 5 \%$ & $\sim 7 \%$ \\
\hline$M L L-A F 6$ & $<1 \%$ & $\sim 3 \%$ & $<1 \%$ & $\sim 7 \%$ & $\sim 13 \%$ & $\sim 5 \%$ \\
\hline$M L L-E L L$ & $<1 \%$ & $<1 \%$ & $<1 \%$ & $\sim 12 \%$ & $\sim 10 \%$ & $\sim 4 \%$ \\
\hline Other $M L L$ & $\sim 9 \%$ & $\sim 8 \%$ & $\sim 1 \%$ & $\sim 22 \%$ & $\sim 34 \%$ & $\sim 15 \%$ \\
\hline
\end{tabular}

a Frequencies are derived from Jansen et al., 2007; and Meyer et al., 2009 (Ref. [33,34]).

b Overall frequency of $M L L$ gene translocation $\left(M L L^{+}\right)$per age group and per disease group.

c Relative frequency of each type of $M L L$ fusion gene within the group of $M L L^{+}$leukemias. 


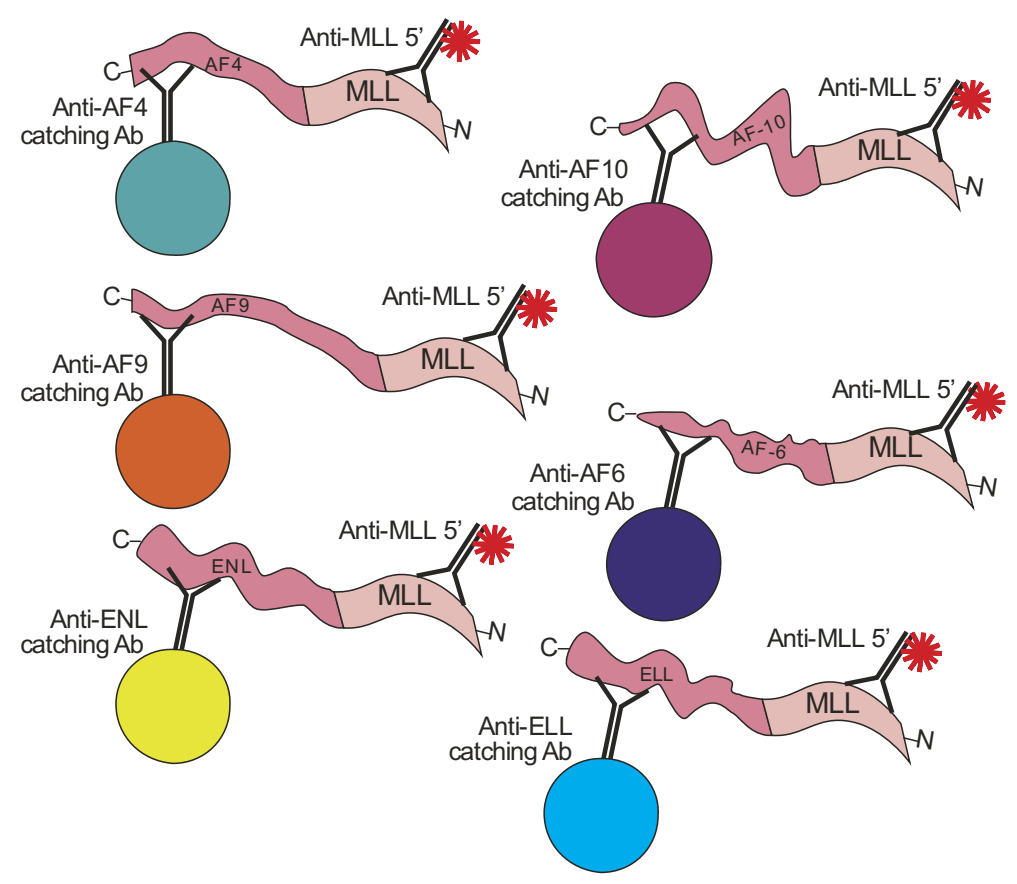

Fig. 7. Proposal for the multiplex MLL fusion protein tube for the detection of the six most frequent fusion proteins. Six differently labeled beads are each coupled to different catching antibodies against a frequently occurring MLL partner, i.e. anti-AF4, anti-AF9, anti-ENL, antiAF10, anti-AF6, or anti-ELL (see Table 3). The PE-conjugated anti-MLL antibody is used as detection antibody for all six beads. This multiplex immunobead assay can detect in a single step the most frequently occurring MLL fusion proteins in $M L L^{+}$acute leukemias.

Factor tube for the detection of AML1-ETO and CBFB-MYH11. In the design of these two multiplex tubes, the incubation and lysis conditions of the singleplex tubes needed adaptations to allow the reliable detection of all relevant fusion proteins. The BCP-ALL multiplex tube and the AML Core Binding Factor tube should be used in addition to the earlier designed BCR-ABL and the PML-RARA tube, respectively.

The multiplex AML Core Binding Factor tube is indeed capable of detecting the two core-bindingfactor fusion proteins AML1-ETO and CBFB-MYH11. The first results of the AML Core Binding Factor tube in more than 60 acute leukemia patients were excellent: full concordance was found between the multiplex immunobead assay and the PCR analyses for AML1-ETO and CBFB-MYH11 transcripts (Table 2) (E. Dekking et al, manuscript in preparation).

The multiplex BCP-ALL tube is meant to detect three different types of fusion proteins of cytoplasmic or nuclear origin, implying that the incubation and lysis conditions needed to be adapted to guarantee efficient detection of all fusion proteins. The preliminary results of the BCP-ALL multiplex assay look promising. However, a large-scale EuroFlow study on a variety of patient samples still needs to be performed (Table 2).

\section{Future immunobead assay: multiplex MLL tube}

We are in the process of extending our efforts toward a multiplex MLL tube, which can detect fusion proteins derived from the most frequently occurring $M L L$ gene translocations.

The MLL gene on chromosome 11q23 can translocate to many different partner genes [1]. Thus far, at least 64 different $M L L$ partner genes have been identified in acute leukemia patients [33,34]. Despite this exceptionally high number of different partner genes, only six different partner genes are found in $\sim 85 \%$ of acute leukemias with an MLL fusion gene: AF4, AF9, ENL, AF10, AF6 and ELL [34]. The relative distribution of these six partner genes differs between ALL and AML and among different age groups 
(Table 3) [33,34]. Also, the prognosis of these $M L L$ gene aberrations ranges from mainly poor for most $M L L$ gene aberrations to fairly good for, for instance, MLL-AF9 in AML. Therefore, it is important to identify the particular $M L L$ gene aberration in an acute leukemia patient. Moreover, in infant ALL the presence of $M L L$ gene rearrangements is the most important prognostic factor associated with treatment failure [35]. Therefore, we now aim at the development of a multiplex MLL tube which can identify six different MLL fusion proteins in a single step (Fig. 7). We realize that the design and development of this MLL multiplex tube will be a challenge, but we believe that that such tool will be important for optimal patient care.

\section{Conclusions}

The flow cytometric immunobead assay for the detection of fusion proteins in lysates of leukemic cells has several advantages over the currently used molecular and genetic techniques:

- easy and reliable technique for fusion protein detection;

- independent of breakpoint position in the involved genes (if antibodies were designed correctly against "outer" epitopes);

- results within 3-4 h;

- multiplex possibilities by using differential labeling of the beads (e.g., different color or different size);

- no need for special laboratory facilities, other than a routine flow cytometer;

- can be run in parallel to standard flow cytometric immunophenotyping.

When using the immunobead assay, two caveats should be considered. The first relates to the effects of protein degradation via protease activity, which need to be prevented by the careful performance of the (pre)incubation and lysis steps with protease inhibitors. The second concerns the sensitivity of the immunobead assay, which varies between $1 \%$ and $10 \%$ and, therefore, does not reach the sensitivity of PCR techniques, but matches that of FISH and cytogenetics.

Overall, we conclude that the flow cytometric immunobead assay for the detection of fusion proteins contributes to a fast and easy diagnosis and classification of leukemias. The abovementioned advantages make the immunobead assay a technically attractive alternative to molecular genetic diagnostics, particularly in diagnostic centers that do not have direct access to molecular laboratory facilities.

The presented progress in the detection of genetic aberrations at the protein level is based on combined efforts of academia and industry. The combination of university (Erasmus MC), spin-off companies (e.g., Dynomics), industry (BD Biosciences), and a clinical-diagnostic network (EuroFlow) provided the complete pathway from invention and development to production and clinical testing of an innovative diagnostic product.

\section{Practice point}

1. The flow cytometric detection of leukemia-specific products of fusion genes is an enormous step in the diagnosis of leukemia patients.

2. The antibody-based immunobead assay allows the easy, rapid and reliable detection of fusion proteins in lieu of time-consuming molecular techniques.

3. Given that BCR-ABL proteins are now detected within 4 hours post sample receipt, offers the opportunity to establish BCR-ABL status in parallel to the immunophenotype of the leukemic blasts and in time to determine patient eligibility for treatment trials.

4. The $1 \%$ sensitivity level of the BCR-ABL immunobead assay, though not suitable for minimal residual disease monitoring, is sufficient to detect $B C R-A B L^{+}$leukemias at diagnosis. 


\section{Research agenda}

1. To date, only the BCR-ABL immunobead assay has become commercially available. Commercial kits for other fusion proteins are either nearing completion (e.g., PML-RARA) or are in development.

2. Multiplexing of immunobeads for a variety of related fusion proteins, either related by disease (TEL-AML1 plus E2A-PBX1 plus MLL-AF4) or related by transcript (e.g., core-binding-factor tube), if successful, would further speed up the molecular diagnosis of a leukemia patient.

3. Problems with protease-mediated degradation of fusion proteins, particularly in the presence of mature myeloid cells, will need to be addressed further. Especially in bone marrow samples with low levels of leukemic blasts. This problem is preferably addressed by inclusion of a control bead, which detects an ubiquously expressed protein.

\section{Conflict of interest statement}

E. Dekking is employee of Dynomics, a spin-off company of Erasmus MC, Rotterdam, The Netherlands; JJM van Dongen and VHJ van der Velden are inventors of the immunobead assay (patent PCT/NL01/00.945), which has been licensed by Erasmus MC to Dynomics; FJT Staal en JJM van Dongen are stockholders of Dynomics and advised BD Bioscienses in the development and optimization of the immunobead assay.

\section{Acknowledgements}

The research and development of the flow cytometric immunobead assay and the EuroFlow Consortium were supported by the European Commission (grant STREP EU-FP6, LSHB-CT-2006018708). We are grateful to Dr. Jean-Luc Sanne of the European Commission for his support and monitoring of the EuroFlow project. We thank Dr. Charlene Bush-Donovan, Dr. Hobert Wai, Dr. Eric Dixon, Dr. Frans Nauwelaers, Dr. Mark Herberger, and Dr. Vera Imper (BD Biosciences) for their technical support in the design of seven of the fourteen applied antibodies and for the production of the various immunobead reagents.

\section{References}

*[1] Mitelman F, Johansson B, Mertens F. The impact of translocations and gene fusions on cancer causation. Nat Rev Cancer 2007;7:233-45.

[2] Rowley JD. Chromosomal translocations: revisited yet again. Blood 2008;112:2183-9.

*[3] Swerdlow SH, Campo E, Harris NL, Jaffe ES, Pileri SA, Stein H, Thiele J, Vardiman JW, editors. WHO classification of tumours of haematopoietic and lymphoid tissues. 4th ed. Lyon: International Agency for Research on Cancer (IARC); 2008.

*[4] de Klein A, van Kessel AG, Grosveld G, et al. A cellular oncogene is translocated to the Philadelphia chromosome in chronic myelocytic leukaemia. Nature 1982;300:765-7.

[5] Bartram CR, de Klein A, Hagemeijer A, et al. Translocation of c-ab1 oncogene correlates with the presence of a Philadelphia chromosome in chronic myelocytic leukaemia. Nature 1983;306:277-80.

[6] Hermans A, Heisterkamp N, von Linden M, et al. Unique fusion of bcr and c-abl genes in Philadelphia chromosome positive acute lymphoblastic leukemia. Cell 1987;51:33-40.

*[7] van Dongen JJ, Macintyre EA, Gabert JA, et al. Standardized RT-PCR analysis of fusion gene transcripts from chromosome aberrations in acute leukemia for detection of minimal residual disease. Report of the BIOMED-1 Concerted Action: investigation of minimal residual disease in acute leukemia. Leukemia 1999;13:1901-28.

[8] Grimwade D, Biondi A, Mozziconacci MJ, et al. Characterization of acute promyelocytic leukemia cases lacking the classic t(15;17): results of the European Working Party. Groupe Français de Cytogénétique Hématologique, Groupe de Français d'Hematologie Cellulaire, UK Cancer Cytogenetics Group and BIOMED 1 European Community-Concerted Action “Molecular Cytogenetic Diagnosis in Haematological Malignancies". Blood 2000;96:1297-308.

*[9] Szczepański T, Harrison CJ, Van Dongen JJM. Genetic aberations in pediactric acute leukemias and implication for patient management. Lancet Oncol 2010;11:880-9.

[10] Anastasiadou E, Schwaller J. Role of constitutively activated protein tyrosine kinases in malignant myeloproliferative disorders: an update. Curr Opin Hematol 2003;10:40-8.

[11] Druker BJ, Talpaz M, Resta DJ, et al. Efficacy and safety of a specific inhibitor of the BCR-ABL tyrosine kinase in chronic myeloid leukemia. N Engl J Med 2001;344:1031-7. 
[12] Schiffer CA. BCR-ABL tyrosine kinase inhibitors for chronic myelogenous leukemia. N Engl J Med 2007;357:258-65.

[13] Weisberg E, Manley PW, Cowan-Jacob SW, et al. Second generation inhibitors of BCR-ABL for the treatment of imatinibresistant chronic myeloid leukaemia. Nat Rev Cancer 2007;7:345-56.

[14] Sanz MA, Grimwade D, Tallman MS, et al. Management of acute promyelocytic leukemia: recommendations from an expert panel on behalf of the European Leukemia Net. Blood 2009;113:1875-91.

[15] Tallman MS, Altman JK. How I treat acute promyelocytic leukemia. Blood 2009;114:5126-35.

*[16] van der Burg M, Poulsen TS, Hunger SP, et al. Split-signal FISH for detection of chromosome aberrations in acute lymphoblastic leukemia. Leukemia 2004;18:895-908.

[17] Gabert J, Beillard E, van der Velden VH, et al. Standardization and quality control studies of 'real-time' quantitative reverse transcriptase polymerase chain reaction of fusion gene transcripts for residual disease detection in leukemia a Europe Against Cancer program. Leukemia 2003;17:2318-57.

*[18] Van Denderen J, Hermans A, Meeuwsen T, et al. Antibody recognition of the tumor-specific bcr-abl joining region in chronic myeloid leukemia. J Exp Med 1989;169:87-98.

[19] van Denderen J, van der Plas D, Meeuwsen T, et al. Immunologic characterization of the tumor-specific bcr-abl junction in Philadelphia chromosome-positive acute lymphoblastic leukemia. Blood 1990;76:136-41

[20] van Denderen J, ten Hacken P, Berendes P, et al. Antibody recognition of the tumor-specific b3-a2 junction of bcr-abl chimeric proteins in Philadelphia-chromosome-positive leukemias. Leukemia 1992;6:1107-12.

[21] van Denderen J, ten Hacken P, Berendes P, et al. Recognition of the ALL-specific BCR-ABL junction in P190bcr-abl by monoclonal antibody ER-FP1. Leukemia 1994;8:1503-9.

[22] Berendes PB, Veenman JN, Van Denderen AC, et al. Recognition of tumor-specific gene products in cancer (Fusion protein dipstick patent). International patent 1998; PCT/NL98/00289.

[23] Berendes PB, Veenman JN, Van Denderen AC, et al. The BCR-ABL dipstick assay: a simple and rapid method for detecting $\mathrm{Ph}+$ leukemias. In: Berendes P, editor. Recognition of tumor-specific proteins in human cancer. Ph.D. Thesis. Rotterdam, The Netherlands: Department of Immunology, Erasmus University Rotterdam; 1997, p. 111-27.

*[24] Van Dongen JJM, Van der Velden VHJ, Berendes PB, et al. Recognition of tumour-specific gene products in cancer (Fusion protein bead patent). International Patent 2001; PCT/NL01/00.945.

[25] Pane F, Frigeri F, Sindona M, et al. Neutrophilic-chronic myeloid leukemia: a distinct disease with a specific molecular marker (BCR/ABL with C3/A2 junction). Blood 1996;88:2410-4.

[26] Melo JV. The diversity of BCR-ABL fusion proteins and their relationship to leukemia phenotype. Blood 1996;88:2375-84.

[27] Boeckx N, Jansen MW, Haskovec C, et al. Identification of e19a2 BCR-ABL fusions (mu-BCR breakpoints) at the DNA level by ligation-mediated PCR. Leukemia 2005;19:1292-5.

[28] Hochhaus A, Reiter A, Skladny H, et al. A novel BCR-ABL fusion gene (e6a2) in a patient with Philadelphia chromosomenegative chronic myelogenous leukemia. Blood 1996;88:2236-40.

*[29] Weerkamp F, Dekking E, Ng YY, et al. Euroflow consortium. Flow cytometric immunobead assay for the detection of BCRABL fusion proteins in leukemia patients. Leukemia 2009;23:1106-17.

[30] van der Velden VH, Beverloo HB, Hoogeveen PG, et al. A novel BCR-ABL fusion transcript (e18a2) in a child with chronic myeloid leukemia. Leukemia 2007;21:833-5.

[31] Stein E, McMahon B, Kwaan H, et al. The coagulopathy of acute promyelocytic leukaemia revisited. Best Pract Res Clin Haematol 2009;22:153-63.

[32] Liso V, Bennett J. Morphological and cytochemical characteristics of leukaemic promyelocytes. Best Pract Res Clin Haematol 2003;16:349-55.

[33] Jansen MW, Corral L, Van der Velden VHJ, et al. Immunobiological diversity in infant acute lymphoblastic leukemia is related to the occurrence and type of MLL gene rearrangement. Leukemia 2007;21:633-41.

*[34] Meyer C, Kowarz E, Hofmann J, et al. New insights to the MLL recombinome of acute leukemias. Leukemia 2009; 23:1490-9.

[35] Pieters R, Schrappe M, De Lorenzo P, et al. A treatment protocol for infants younger than 1 year with acute lymphoblastic leukaemia (Interfant-99): an observational study and a multicentre randomised trial. Lancet 2007;370:240-50. 